\title{
13.2 Автоматизация предполетного информационного обеспечения
}

\section{Введение}

На каждом аэродроме, который используется для международных полетов, персоналу, связанному с выполнением полетов, включая летный экипаж и службы, ответственные за предполетную информацию, предоставляется аэронавигационная информация, необходимая для обеспечения безопасности, регулярности и эффективности полетов, которая касается всех этапов маршрута, начиная с данного аэродрома. Предполетная информация включает в себя много вопросов таких, как информация о маршруте полета, об аэродроме назначения и запасном аэродроме (3А), о правилах загрузки воздушного судна (BC), анализ минимумов для посадки на аэродром назначения и запасной, анализ метеорологической обстановки по маршруту полета, правила расчета топлива для различных видов и условий полета и др. [622].

В соответствии с установленными правилами каждому полету должна предшествовать тщательная подготовка. Перед каждым полетом, экипажи ВС (ЭВС) на предварительной подготовке должны быть обеспечены всей необходимой предполетной информацией, в результате изучения которой ЭВС выбирает 3А и принимает решение о возможности выполнения полета [622].

На сегодняшний день информация, которая влияет на выбор 3А - величина детерминированная $[623,624]$. Основными обязанностями сотрудника по обеспечению полетов/летного диспетчера (FOO/FD) есть осуществление квалифицированной помощи командиру воздушного судна (КВС) по подготовке рабочего плана полета и обеспечения КВС в полете информацией при помощи соответствующих средств.

В целях оптимизации предполетной подготовки в авиации разрабатываются и применяются автоматизированные системы аэронавигационной информации (АС ППИ) [625]. 


\section{Анализ исследований и публикаций}

Предполетная подготовка ЭВС является одним из условий обеспечения безопасности полетов. Перед каждым полетом, ЭВС на предполетной подготовке необходимо выбрать ЗА, а затем, принимая решение на вылет, уточнить метеоусловия на них. Выбор ЗА и принятие решения на вылет регламентируют следующие документы: Руководство по летной эксплуатации $\mathrm{BC}$, Наставление по производству полетов гражданской авиации, и принятый в 2005 году Государственной авиационной службой Украины «Порядок принятия решения на вылет и прилет воздушных судов гражданской авиации Украины по правилам полета по приборам».

В настоящее время в целях повышения безопасности полетов в состав автоматизированных систем управления воздушным движением (АС УВД) включаются интеллектуальные модули - СППР, в которых реализована информационная поддержка авиационного оператора (AO). Они содействуют оперативному принятию решения экипажем ВС. АС УВД с элементами информационной поддержки, используемые в авиации, представлены в работах [626-629].

Элементы СППР включены в состав АС УВД - «Alenia» (аэропорт Борисполь), «Ксения», «Настасия», «Юлия» (Львов, Симферополь, Харьков, Одесса), «Альфа» (Россия), «Тотрson» (Узбекистан) и другие, где выполняют функции информационной поддержки принятия решения авиационным оператором. Однако в службах предполетного информационного обслуживания СППР не использовались.

В целях предполетного обслуживания и обеспечения полета эксплуатанты используют ряд программных продуктов. Наиболее известными поставщиками указанных программ являются фирмы Jeppesen, SITA, Sabre, Skyplan, Lido и ряд других. Некоторые авиакомпании используют программное обеспечение собственной разработки [622]. Использование таких программ позволяет осуществлять аэронавигационное и метеорологическое обеспечение полётов. 
Однако автоматически сгенерированный компьютером оперативный план полёта не всегда является рациональным для выполнения данного рейса. Компьютер обычно подбирает наиболее короткий подходящий маршрут, не учитывая множества других эксплуатационных факторов, влияющих на выполнение полёта. Подбор компьютером не самой подходящей с экономической точки зрения высоты полёта может привести к значительному перерасходу топлива, особенно на длинных рейсах [622].

Алгоритм выбора запасного аэродрома в программе планирования полета производится путем отбора из встроенной базы данных аэропортов при вводе пользователем дополнительных параметров выбора (например, удаления от аэропорта назначения, данные по топливу). Данный недостаток таких систем затрудняет процесс выбора запасного аэродрома на этапе планирования полета, так как авиационному оператору затруднительно самостоятельно сделать объективный выбор наиболее подходящих запасных аэродромов по маршруту полета, тем более, в таких программах не производится анализ метеоусловий на запасных аэродромах. Также в таких программах не реализована информационная поддержка принятия решения о выполнении полета по маршруту.

\section{Постановка задачи}

1. Разработка модуля автоматизированного выбора запасного аэродрома.

2. Построение нейросетевой модели анализа возможности выполнения полета.

\section{Разработка модуля автоматизированного выбора запасного аэродрома}

Центральное место в системе предполетной подготовки занимает проблема выбора оптимального ЗА по маршруту полета и задача принятия решения относительно возможности выполнения полета. Для решения этой задачи в таких системах предлагается применение модуля автоматизированного выбора запасного аэродрома [630]. 
Чтобы оптимизировать решение задачи выбора ЗА и добиться более точных и объективных результатов, предлагается поэтапный подход к методике выбора 3А. Рассмотрим работу модуля.

Первый этап. Ввод полетного задания.

Он заключается во вводе пользователем полетного задания в расчетную систему, т.е. ввод аэродрома (АД) вылета и назначения [631].

Данные о выбранных аэродромах и сведения по выбранному маршруту поступают в модуль выбора ЗА из базы данных.

Второй этап. Определение потенциально возможных запасных аэродромов.

В блоке определения возможных ЗА на основе введенного полетного задания происходит определение маршрута и определение возможных запасных аэродромов. Сначала определяется расстояние от аэродрома вылета до аэродрома назначения $\mathrm{L}_{\text {маршрута }}$ и в радиусе $\mathrm{R}$ равном половине маршрута $\mathrm{c}$ центром в аэродроме назначения определятся возможные запасные аэродромы. Далее происходит сортировка возможных запасных аэродромов в порядке возрастания расстояния от аэродрома назначения до них.

Третий этап. Первое оценивание аэродромов по факторам и отсев заранее непригодных аэродромов.

Выбранные возможные аэродромы оцениваются по 3-м значимым факторам:

1. А - Тактико-технические характеристики аэродрома (характеристики взлетно-посадочной полосы, светотехнического оборудования, системы захода на посадку, навигационной система подхода).

2. $\mathrm{B}-$ Метеоусловия.

3. $\mathrm{C}-$ Количество топлива.

Оценивание аэродромов по всем факторам будем осуществлять с помощью двухслойной прямонаправленной нейронной сети - двухслойного персептрона [632]. Входными параметрами модели являются факторы, которые влияют на выбор запасного аэродрома при принятии решения на вылет: 
- компонент $\bar{A}$ - оценка тактико - технических характеристик 3А, (a 1 - TTX ВПП, $\mathrm{a}_{2}$ - система захода на посадку, $\mathrm{a}_{3}$ - навигационная система подхода, $\mathrm{a}_{4}$ светотехническое оборудование) $\overline{\mathrm{A}}=\left\{a_{i}\right\}, i=\overline{1,4}$;

- компонент $\bar{B}$ - оценка метеоусловий на предполагаемом 3А (b соответствие НГО и видимости установленным минимумам, $\mathrm{b}_{2}$ - соответствие составляющих ветра установленным предельно допустимым), $\overline{\mathrm{B}}=\left\{b_{j}\right\}, j=\overline{1,2}$.

- компонент $\bar{C}$ - оценка наличия топлива на борту ВС, т.е. хватит ли топлива до предполагаемого ЗА, $\overline{\mathrm{C}}=\left\{c_{k}\right\}, k=\overline{1,1}$;

Каждому входному параметру ставится также бинарный вектор, который отображает соответствие (1) или несоответствие (0) определенного параметра удовлетворительным условиям.

Выходным параметром модели является оценка аэродромов относительно возможности быть выбранными в качестве запасных $\overline{\mathrm{R}}$ : $\mathrm{r} 1$ - АД может быть запасным (1), или АД не может быть запасным (0), $\overline{\mathrm{R}}=\left\{r_{x}\right\}, x=\overline{1,1}$.

Таким образом, определяются аэродромы, которые могут быть выбраны в качестве запасных.

Четвертый этап. Второе оценивание аэродромов по факторам.

На четвертом этапе аэродромы, которые могут быть выбраны в качестве запасных, оцениваются с точки зрения экономичности. Здесь играют роль такие факторы как аэронавигационные сборы по маршруту и на аэродроме, количество топлива, маршрут и другие факторы.

Выбранные пригодные аэродромы оцениваются по данным факторам и выбирается оптимальный запасной аэродром.

Данные о выбранном запасном аэродроме поступают на интерфейс пользователя. 


\section{Построение нейросетевой модели анализа возможности выполнения} полета

Анализ возможности выполнения полета в автоматизированной системе подготовки предполетной информации осуществляется с помощью трехслойной прямонаправленной нейронной сети [632] (Рис. 1).

Входными параметрами первого слоя модели являются исходы, анализирующие состояние подфакторов (Табл. 1). В соответствии с каждым входным параметром ставится бинарный вектор, который отображает результат состояния исходов: соответствие (1) или несоответствие (0) требуемым условиям определенного подфактора. Выходы первого слоя являются входными параметрами второго слоя и отображают состояние факторов (Табл.2).

Бинарный вектор отображает оценку состояния фактора: удовлетворяют ли они требуемым условиям для выполнения полета: 1 - факторы соответствуют, 0 - факторы не соответствуют.

Таблица 1

Таблица критериев (оценка состояния подфакторов)

\begin{tabular}{|c|c|c|c|c|}
\hline Факторы & Критерий оценки & Исход & $\begin{array}{l}\text { Результа } \\
\text { т исхода }\end{array}$ & Комментарии \\
\hline \multirow{6}{*}{$\mathbf{A}$} & $\mathrm{A}=\left(\mathrm{a}_{\mathrm{adm} 1}-\mathrm{a}_{1}\right) \geq 0$ & \multirow[t]{2}{*}{$\mathrm{A}_{1}$} & $\mathrm{~A} 1=1$ & $\begin{array}{lrr}\text { Взлетная } & \text { масса } \\
\text { превышает } & \text { пр } \\
\text { допустимую } & \\
\end{array}$ \\
\hline & $A=\left(a_{a d m 1}-a_{1}\right)<0$ & & $\mathrm{~A} 2=0$ & $\begin{array}{l}\text { Взлетная масса превышает } \\
\text { предельно допустимую }\end{array}$ \\
\hline & $\mathrm{A}=\left(\mathrm{a}_{\mathrm{adm} 2}-\mathrm{a}_{2}\right) \geq 0$ & \multirow{2}{*}{$\mathrm{A}_{2}$} & $\mathrm{~A} 3=1$ & $\begin{array}{l}\text { Посадочная масса не } \\
\text { превышает допустимую }\end{array}$ \\
\hline & $\mathrm{A}=\left(\mathrm{a}_{\mathrm{adm} 2}-\mathrm{a}_{2}\right)<0$ & & $\mathrm{~A} 4=0$ & $\begin{array}{l}\text { Посадочная масса } \\
\text { превышает допустимую }\end{array}$ \\
\hline & $\mathrm{A}=\left(\mathrm{a}_{\mathrm{adm} 1}-\mathrm{a}_{1}\right) \geq 0$ & \multirow{2}{*}{$\mathrm{A}_{3}$} & $\mathrm{~A} 5=1$ & $\begin{array}{l}\text { Центровка ВС в пределах } \\
\text { нормы }\end{array}$ \\
\hline & $\mathrm{A}=\left(\mathrm{a}_{\mathrm{adm} 1}-\mathrm{a}_{1}\right)<0$ & & $\mathrm{~A} 6=0$ & $\begin{array}{l}\text { Центровка } \\
\text { нормых }\end{array}$ \\
\hline \multirow{3}{*}{ B } & $\begin{aligned} b_{2}= & \left(b_{1} \leq b_{21}\right) \wedge\left(b_{1} \leq b_{22}\right) \wedge \\
& \wedge\left(b_{1} \leq b_{23}\right)\end{aligned}$ & \multirow[b]{2}{*}{$\mathrm{B}_{1}$} & ${ }_{\mathrm{B} 1=} \Pi_{1}$ & $\begin{array}{l}\text { одолжение Таблицы } 1 \\
\text { минимумам }\end{array}$ \\
\hline & иначе & & $\mathrm{B} 2=0$ & $\begin{array}{ll}\left(\mathrm{H}_{\text {нго }} \times \mathrm{RVR}\right) & \text { не } \\
\text { соответствует } & \\
\text { установленным } & \\
\text { минимумам } & \end{array}$ \\
\hline & $\begin{array}{l}\mathrm{b}_{4}=\left(\mathrm{b}_{41}-\mathrm{b}_{3}\right) \geq 0 \text { при } \quad \text { табличном } \\
\text { значении } \mathrm{b}_{42}\end{array}$ & $\mathrm{~B}_{2}$ & $\mathrm{~B} 3=1$ & $\begin{array}{ll}\text { боковая } & \text { составляющая } \\
\text { ветра }\left(\mathrm{U}_{\sigma}\right) & \text { соответствует } \\
\text { предельно допустимой }\end{array}$ \\
\hline
\end{tabular}


Продолжение Таблицы 1

\begin{tabular}{|c|c|c|c|c|}
\hline Факторы & Критерий оценки & Исход & $\begin{array}{l}\text { Результа } \\
\text { т исхода }\end{array}$ & Комментарии \\
\hline & $\begin{array}{l}\mathrm{b}_{4}=\left(\mathrm{b}_{41}-\mathrm{b}_{3}\right)<0 \text { при табличном } \\
\text { значении } \mathrm{b}_{42}\end{array}$ & & $\mathrm{~B} 4=0$ & $\begin{array}{l}\mathrm{U}_{\sigma} \text { не } \quad \text { соответствует } \\
\text { предельно допустимой }\end{array}$ \\
\hline \multirow[b]{2}{*}{$\mathbf{C}$} & $\left(c_{1}=0\right) \wedge\left(c_{2}=0\right) \wedge \ldots \wedge\left(c_{9}=\right.$ & \multirow[b]{2}{*}{$\mathrm{C}_{1}$} & $\mathrm{C} 1=1$ & все ОМЯ отсутствуют \\
\hline & иначе & & $\mathrm{C} 2=0$ & $\begin{array}{l}\text { присутствует хотя бы одно } \\
\text { ОМЯ }\end{array}$ \\
\hline \multirow{4}{*}{$\mathbf{D}$} & $\begin{aligned} d_{2}= & \left(d_{1} \leq d_{21}\right) \wedge\left(d_{1} \leq d_{22}\right) \wedge \\
& \wedge\left(d_{1} \leq d_{23}\right)\end{aligned}$ & \multirow[b]{2}{*}{$\mathrm{D}_{1}$} & $\mathrm{D} 1=1$ & $\begin{array}{l}\left(\mathrm{H}_{\text {нго }} \times \mathrm{RVR}\right) \text { соответствует } \\
\text { установленным } \\
\text { минимумам }\end{array}$ \\
\hline & иначе & & $\mathrm{D} 2=0$ & $\begin{array}{ll}\left(\mathrm{H}_{\text {нго }} \times \mathrm{RVR}\right) & \text { не } \\
\text { соответствует } & \\
\text { установленным } & \\
\text { минимумам } & \end{array}$ \\
\hline & $\begin{array}{l}\mathrm{d}_{4}=\left(\mathrm{d}_{41}-\mathrm{d}_{3}\right) \geq 0 \text { при } \quad \text { табличном } \\
\text { значении } \mathrm{d}_{42}\end{array}$ & \multirow{2}{*}{$\mathrm{D}_{2}$} & $\mathrm{D} 3=1$ & $\begin{array}{l}\mathrm{U}_{\sigma} \quad \text { соответствует } \\
\text { предельно допустимой }\end{array}$ \\
\hline & $\begin{array}{l}\mathrm{d}_{4}=\left(\mathrm{d}_{41}-\mathrm{d}_{3}\right)<0 \text { при } \\
\text { значении табичном } \mathrm{d}_{42}\end{array}$ & & $\mathrm{D} 4=0$ & $\begin{array}{l}\mathrm{U}_{6} \quad \text { не } \quad \text { соответствует } \\
\text { предельно допустимой }\end{array}$ \\
\hline \multirow{4}{*}{$\mathbf{E}$} & $\begin{aligned} e_{2}= & \left(e_{1} \leq d_{21}\right) \wedge\left(e_{1} \leq d_{22}\right) \wedge \\
& \wedge\left(e_{1} \leq d_{23}\right)\end{aligned}$ & \multirow[b]{2}{*}{$\mathrm{E}_{1}$} & $\mathrm{E} 1=1$ & $\begin{array}{l}\left(\mathrm{H}_{\text {нго }} \times \mathrm{RVR}\right) \text { соответствует } \\
\text { установленным } \\
\text { минимумам }\end{array}$ \\
\hline & иначе & & $\mathrm{E} 2=0$ & $\begin{array}{ll}\left(\mathrm{H}_{\text {Hго }} \times \mathrm{RVR}\right) & \text { не } \\
\text { соответствует } & \\
\text { установленным } & \\
\text { минимумам } & \\
\end{array}$ \\
\hline & $\begin{array}{l}\mathrm{e}_{4}=\left(\mathrm{d}_{41}-\mathrm{e}_{3}\right) \geq 0 \quad \text { при } \\
\text { значении } \mathrm{d}_{42}\end{array}$ & \multirow{2}{*}{$\mathrm{E}_{2}$} & $\mathrm{E} 3=1$ & $\begin{array}{l}\mathrm{U}_{\sigma} \\
\text { предельно допустимой }\end{array}$ \\
\hline & $\begin{array}{l}\mathrm{e}_{4}=\left(\mathrm{d}_{41}-\mathrm{e}_{3}\right)<0 \\
\text { значении } \mathrm{d}_{42}\end{array}$ & & $\mathrm{E} 4=0$ & $\begin{array}{l}\mathrm{U}_{\sigma} \quad \text { не } \quad \text { соответствует } \\
\text { предельно допустимой }\end{array}$ \\
\hline \multirow{2}{*}{$\mathbf{F}$} & $\mathrm{f}_{1}=1$ & \multirow{2}{*}{$\mathrm{F}_{1}$} & $\mathrm{~F} 1=1$ & FPL принят IFPS \\
\hline & $\mathrm{f}_{1}=0$ & & $\mathrm{~F} 2=0$ & FPL не принят IFPS \\
\hline \multirow[t]{2}{*}{$\mathbf{H}$} & $\left(h_{1}=1\right) \wedge\left(h_{2}=1\right) \wedge\left(h_{3}=1\right)$ & \multirow[t]{2}{*}{$\mathrm{H}_{1}$} & $\mathrm{H} 1=1$ & $\begin{array}{l}\text { Состояние АД вылета, } \\
\text { назначения } \\
\text { соответствуют у } \text { Воловиям } \\
\text { вылета, посадки и полета } \\
\text { по маршруту }\end{array}$ \\
\hline & иначе & & $\mathrm{H} 2=0$ & $\begin{array}{l}\text { Состояние } \\
\text { соответствует }\end{array}$ \\
\hline \multirow{2}{*}{$\mathbf{I}$} & $i_{1}=1$ & \multirow{2}{*}{$\mathrm{I}_{1}$} & $\mathrm{I} 1=1$ & ВС к полету готово \\
\hline & $i_{1}=0$ & & $\mathrm{I} 2=0$ & ВС к полету не готово \\
\hline \multirow{2}{*}{$\mathbf{K}$} & $\mathrm{k}_{1}=1$ & \multirow{2}{*}{$\mathrm{K}_{1}$} & $\mathrm{~K} 1=1$ & ЭВС к полету готов \\
\hline & $\mathrm{k}_{1}=0$ & & $\mathrm{~K} 2=0$ & ЭВС к полету не готов \\
\hline \multirow{2}{*}{$\mathbf{L}$} & $l_{1}=1$ & \multirow{2}{*}{$\mathrm{L}_{1}$} & $\mathrm{~L} 1=1$ & 3А выбран \\
\hline & $1_{1}=0$ & & $\mathrm{~L} 2=0$ & 3А не выбран \\
\hline \multirow{4}{*}{$\mathbf{M}$} & $\mathrm{M}_{\text {проф }}=1$ & \multirow{2}{*}{$\mathrm{M}_{1}$} & $\mathrm{M} 1=1$ & ЭВС к полету готов \\
\hline & $\mathrm{M}_{\text {проф }}=0$ & & $\mathrm{M} 2=0$ & ЭВС к полету не готов \\
\hline & $\mathrm{M}_{\text {непроф }}=1$ & \multirow{2}{*}{$\mathrm{M}_{2}$} & $\mathrm{M} 3=1$ & ЭВС к полету готов \\
\hline & $\mathrm{M}_{\text {непроф }}=0$ & & $\mathrm{M} 4=0$ & ЭВС к полету не готов \\
\hline
\end{tabular}

Выходным параметром модели является оценка относительно возможности выполнения полета $\overline{\mathrm{G}}: \mathrm{g} 1$ - полет возможен (1), g2 -полет не возможен (0), $\overline{\mathrm{G}}=\left\{g_{x}\right\}, x=\overline{1,0}$. 
Таблица 2

Таблица критериев (оценка состояния факторов)

\begin{tabular}{|c|c|c|c|}
\hline $\begin{array}{c}\text { Факто } \\
\text { ры }\end{array}$ & Критерий оценки & $\begin{array}{l}\text { Результат } \\
\text { исхода }\end{array}$ & Комментарии \\
\hline \multirow[b]{2}{*}{ A } & $A_{1} \in A 1 \wedge A_{2} \in A 3 \wedge A_{3} \in A 5$ & $A=1$ & $\begin{array}{l}\text { Масса ВС удовлетворяет условиям для } \\
\text { взлета и посадки }\end{array}$ \\
\hline & иначе & $A=0$ & $\begin{array}{l}\text { Масса ВС не удовлетворяет условиям } \\
\text { для взлета и посадки }\end{array}$ \\
\hline \multirow{2}{*}{ B } & $B_{1} \in B 1 \wedge B_{2} \in B 3$ & $B=1$ & $\begin{array}{l}\text { МУ на АДв удовлетворят возможности } \\
\text { вылета }\end{array}$ \\
\hline & иначе & $\mathrm{B}=0$ & $\begin{array}{l}\text { МУ на АДв не } \\
\text { возможности вылета }\end{array}$ \\
\hline \multirow{2}{*}{$\mathrm{C}$} & $C_{1} \in C 1$ & $\mathrm{C}=1$ & все ОМЯ отсутствуют \\
\hline & иначе & $\mathrm{C}=0$ & присутствует хотя бы одно ОМЯ \\
\hline \multirow[b]{2}{*}{ D } & $D_{1} \in D 3 \wedge D_{2} \in D 3$ & $\mathrm{D}=1$ & $\begin{array}{l}\text { Фактические МУ на АДн удовлетворят } \\
\text { возможности вылета }\end{array}$ \\
\hline & иначе & $\mathrm{D}=0$ & $\begin{array}{l}\text { Фактические МУ на АДн } \\
\text { удовлетворят возможности вылета }\end{array}$ \\
\hline \multirow{2}{*}{$\mathbf{E}$} & $E_{1} \in E 3 \wedge E_{2} \in E 3$ & $\mathrm{E}=1$ & $\begin{array}{l}\text { Прогнозируемые } \begin{array}{l}\text { МУ } \\
\text { уда } \\
\text { удовлетворят возможности вылета }\end{array} \\
\end{array}$ \\
\hline & иначе & $\mathrm{E}=0$ & $\begin{array}{l}\text { Прогнозируемые МУ на АДн не } \\
\text { удовлетворят возможности вылета }\end{array}$ \\
\hline \multirow{2}{*}{$\mathbf{F}$} & $F_{1} \in F 1$ & $\mathrm{~F}=1$ & FPL принят IFPS \\
\hline & иначе & $\mathrm{F}=0$ & FPL не принят IFPS \\
\hline \multirow[t]{2}{*}{$\mathbf{H}$} & $H_{1} \in H 1$ & $\mathrm{H}=1$ & $\begin{array}{l}\text { Состояние АД вылета, назначения и } \\
\text { ВО соответствуют условиям вылета, } \\
\text { посадки и полета по маршруту }\end{array}$ \\
\hline & иначе & $\mathrm{H}=0$ & Состояние не соответствует \\
\hline \multirow{2}{*}{ I } & $I_{1} \in I 1$ & $\mathrm{I}=1$ & ВС к полету готово \\
\hline & иначе & $\mathrm{I}=0$ & ВС к полету не готово \\
\hline \multirow[t]{2}{*}{$\mathbf{K}$} & $K_{1} \in K 1$ & $\mathrm{~K}=1$ & ЭВС к полету готов \\
\hline & иначе & $\mathrm{K}=0$ & ЭВС к полету не готов \\
\hline \multirow{2}{*}{$\mathbf{L}$} & $L_{1} \in L 1$ & $\mathrm{~L}=1$ & ЗА выбран \\
\hline & иначе & $\mathrm{L}=0$ & ЗА не выбран \\
\hline \multirow{2}{*}{ M } & $M_{1} \in M 1 \wedge M_{2} \in M 3$ & $\mathrm{M}=1$ & $\begin{array}{l}\text { Профессиональные } \\
\text { непрофессиональные факторы ЭВС в } \\
\text { допустимых пределах }\end{array}$ \\
\hline & иначе & $\mathrm{M}=0$ & $\begin{array}{lr}\text { Профессиональные } & \text { и } \\
\text { непрофессиональные } & \text { факторы } \\
\text { выходят за пределы нормы } & \end{array}$ \\
\hline
\end{tabular}




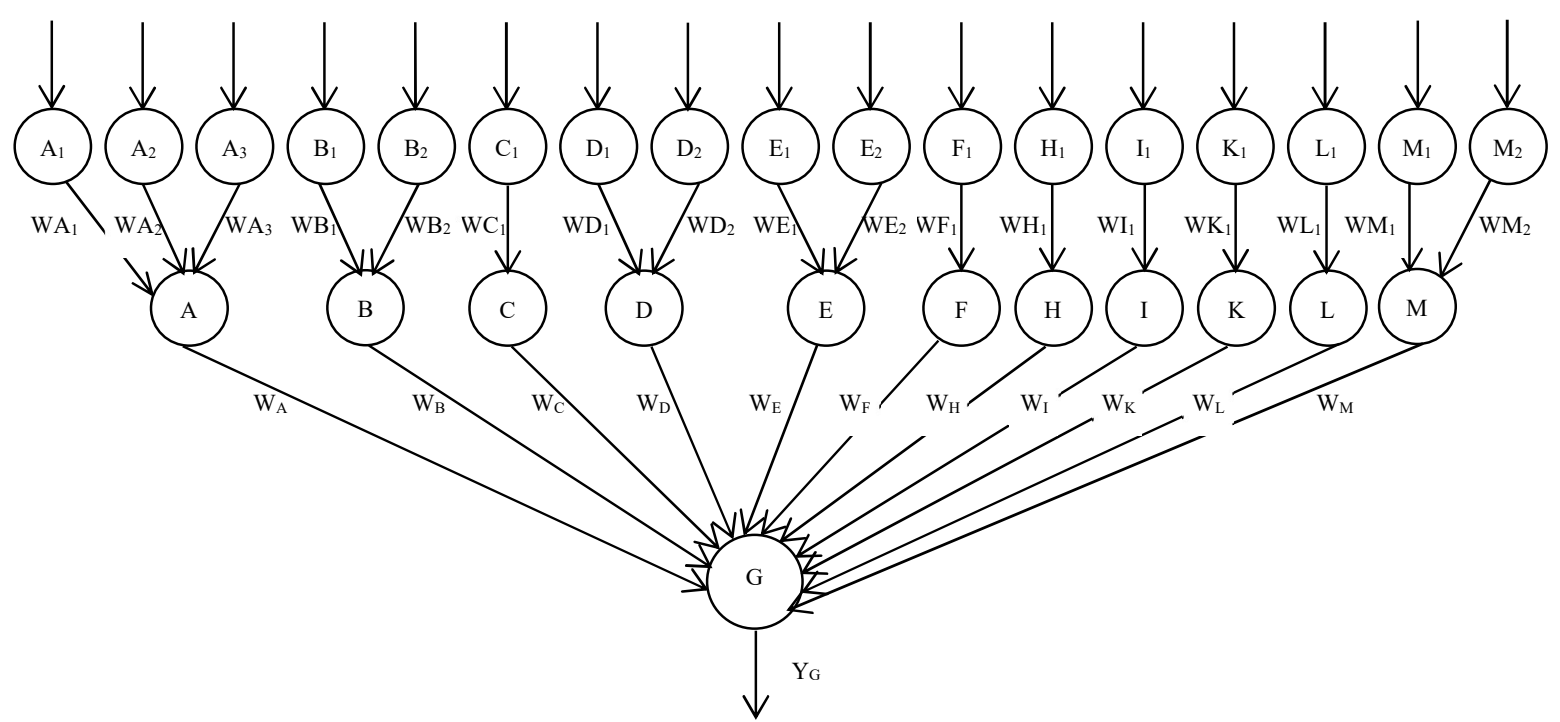

Рисунок 1. - Нейросетевая модель анализа возможности выполнения полета

Разработанная нейросетевая модель позволит оперативно получать командиру ВС рекомендацию о возможности выполнения полета благодаря комплексному учету влияния отдельных факторов. Это сократит время на принятие решения, а также минимизирует возможность принятия неправильного решения.

\section{Выводы}

Рассмотренная проблема выбора запасного аэродрома является актуальной не только в контексте предполетной подготовки. Нередко проблема выбора альтернативного аэродрома или другого места для вынужденной посадки возникает уже в полете. Такие ситуации, требующие вынужденной посадки, всегда связаны с дефицитом времени и высокой нервно-эмоциональной нагрузкой при принятии решения. Таким образом, просматриваются пути внедрения подобных модулей автоматизированного выбора ЗА в бортовых автоматизированных системах управления полетом для определения оптимального запасного аэродрома или пригодного места посадки в любой момент времени и в любой точке маршрута. Таким нововведениям способствуют и существующие и широко применяющиеся в авиации системы глобального позициционирования (GPS); набирающие обороты и все больше применяемые для целей авиации геоинформационные системы (ГИС); а также возможность получать необходимую информацию из баз данных аэропортов или непосредственно из самих аэропортов в режиме on-line. 